\title{
Nicotinamide adenine dinucleotide fluorescence to assess microvascular disturbances in post-COVID-19 patients
}

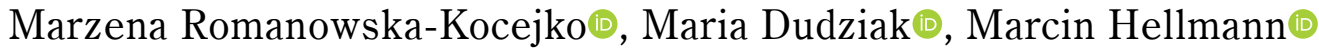 \\ Department of Cardiac Diagnostics, Medical University, Gdansk, Poland
}

Coronavirus disease 2019 (COVID-19) revealed many different faces, from severe to completely asymptomatic course. An increasing number of patients are starting to emerge with significant cardiovascular problems, including myocarditis, heart failure, severe arrhythmias, and thromboembolic complications. Accumulating data suggest that COVID-19 is a systemic vascular endothelial dysfunction with different and unpredictable clinical manifestations, including pulmonary, neurological, cardiac, or thromboembolic problems. Of note, severe acute respiratory syndrome coronavirus 2 (SARS-CoV-2) enters target cells via angiotensin-converting enzyme 2 (ACE2) receptors, which are widely expressed on the endothelial cells in several organs, including the heart and lungs [1]. Indeed, an increasing amount of evidence indicates that SARS-CoV-2 affects endothelial function via inflammation of endothelial cells (endotheliitis), causing microvascular disturbances and microthrombosis in different vascular beds, leading to COVID-19-related acute and long-term complications [2].

More precisely, SARS-CoV-2 affects vascular endothelium by multiple mechanisms, including a cytokine storm. Interestingly, exocytosis of granules from endothelial cells induces platelet aggregation and leukocyte influx into the vessel wall, which causes inflammation, microthrombosis, and capillary obstruction [3]. The blood flow disturbances in capillaries due to endotheliitis or accumulation of leukocytes shortens the time of blood flow resulting in reduced oxygen exchange between blood and tissue. Hypoxia of endothelial cells via transit-time effects leads to the further secretion of inflammatory cytokines, which provides another brick for endothelial damage [4].

Microcirculation plays a pivotal role in tissue oxygenation and nutrient supply. The decrease in oxygen delivery during endotheliitis may induce tissue hypoxia and inhibits metabolism. Accumulating evidence suggests that COVID-19-induced endotheliitis is predominately a systemic small-vessel vasculitis not involving the large arteries such as the main coronaries [5]. Owing to its accessibility, a peripheral microvascular function has been considered an indicator of general microvascular function [6]. Several methods are available to study peripheral microcirculation and attempt to quantify perfusion or oxygenation. However, we can currently assess the variation in tissue biochemistry in vivo using the measurements of a nicotinamide adenine dinucleotide (NADH) fluorescence signal intensity [7].

The newly developed flow-mediated skin fluorescence (FMSF) is a non-invasive optical technique to study microcirculation and metabolic regulation based on cutaneous NADH fluorescence intensity registration. Excitation of the forearm with ultraviolet light at $340 \mathrm{~nm}$ results in the emission of a NADH fluorescence signal from human epidermal cells. Indeed, the epidermis is particularly sensitive to hypoxia. The level of $\mathrm{NADH}$ fluorescence corresponds to the balance of mitochondrial oxidation-reduction processes occurring in the tissue, reflected by the balance between the oxidized form of the coenzyme $\left(\mathrm{NAD}^{+}\right)$

Address for correspondence: Prof. Marcin Hellmann, MD, PhD, Department of Cardiac Diagnostics, Medical University of Gdansk, ul. Smoluchowskiego 17, 80-214 Gdańsk, Poland, tel: +48 58349 33 80, fax: +48 5834933 79, e-mail: marcin.hellmann@gmail.com

Received: 15.09.2021 Accepted: 11.10.2021 Early publication date: 18.10.2021

This article is available in open access under Creative Common Attribution-Non-Commercial-No Derivatives 4.0 International (CC BY-NC-ND 4.0) license, allowing to download articles and share them with others as long as they credit the authors and the publisher, but without permission to change them in any way or use them commercially. 
and its reduced form (NADH). The emitted fluorescence light of NADH at $460 \mathrm{~nm}$ is detected by the receiver diode and corresponds to the activity of microcirculation [7]. Of note, the FMSF device exhibits excellent reproducibility and reasonable agreement for repeated measurements [8].

To assess microvascular reactivity and endothelial function, the FMSF device measures the changes in the intensity of NADH fluorescence in the epidermis over time in response to brachial artery occlusion. Technically, FMSF registers two principal parameters: ischemic response (IR) and hyperemic response (HR). The parameter $\mathrm{IR}_{\max }$ is defined as the ratio (in \%) of relative to maximal baseline increase in NADH fluorescence intensity observed over occlusion, whereas $\mathrm{IR}_{\text {index }}$ is calculated as the area under the curve (AUC) of IR in relation to the baseline. Subsequently, the parameter $\mathrm{HR}_{\max }$ is expressed (in \%) as the relative to maximal baseline decrease in $\mathrm{NADH}$ fluorescence intensity during the reperfusion phase, while $\mathrm{HR}_{\text {index }}$ is defined as the AUC of the IR. When HR reflects microvascular reactivity and endothelial function, the IR may mirror tissue sensitivity to hypoxia (Fig. 1) [9].

Additionally, the FMSF device registers oscillations in the microcirculation, known as flowmotion, specifically present in skin microvascular blood flow. We distinguish endothelial, neurogenic, myogenic, respiratory, and cardiac oscillations based on the frequency analysis. There is compelling evidence that impaired flowmotion may be a symptom of various disorders, including diabetes, a broad spectrum of cardiovascular diseases, and autoimmune and infectious diseases such a COVID-19 [10].

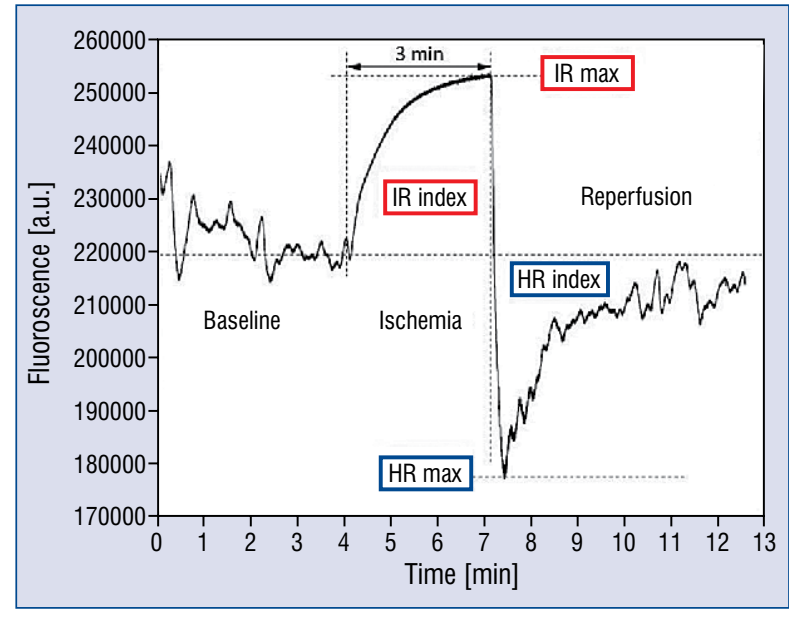

Figure 1. Exemplary image of nicotinamide adenine dinucleotide (NADH) fluorescence trace in response to blockage and release of blood flow in the brachial artery. The ischemic response $\left(I_{\max }\right.$ and $\left.I R_{\text {index }}\right)$ is relative to the baseline increase in NADH fluorescence intensity observed during occlusion, and the hyperemic response $\left(H R_{\text {max }}\right.$ and $\left.H R_{\text {index }}\right)$ is relative to baseline decrease in NADH fluorescence intensity over the reperfusion stage.

The direct measurement of oscillations during the reperfusion stage enables us to determine the hypoxia sensitivity (HS) parameter, which covers the intensity of flowmotion related to myogenic oscillations. Of note, myogenic oscillations are mainly stimulated on the reperfusion line following transient ischemia. Therefore, the HS parameter seems to be particularly significant to determine

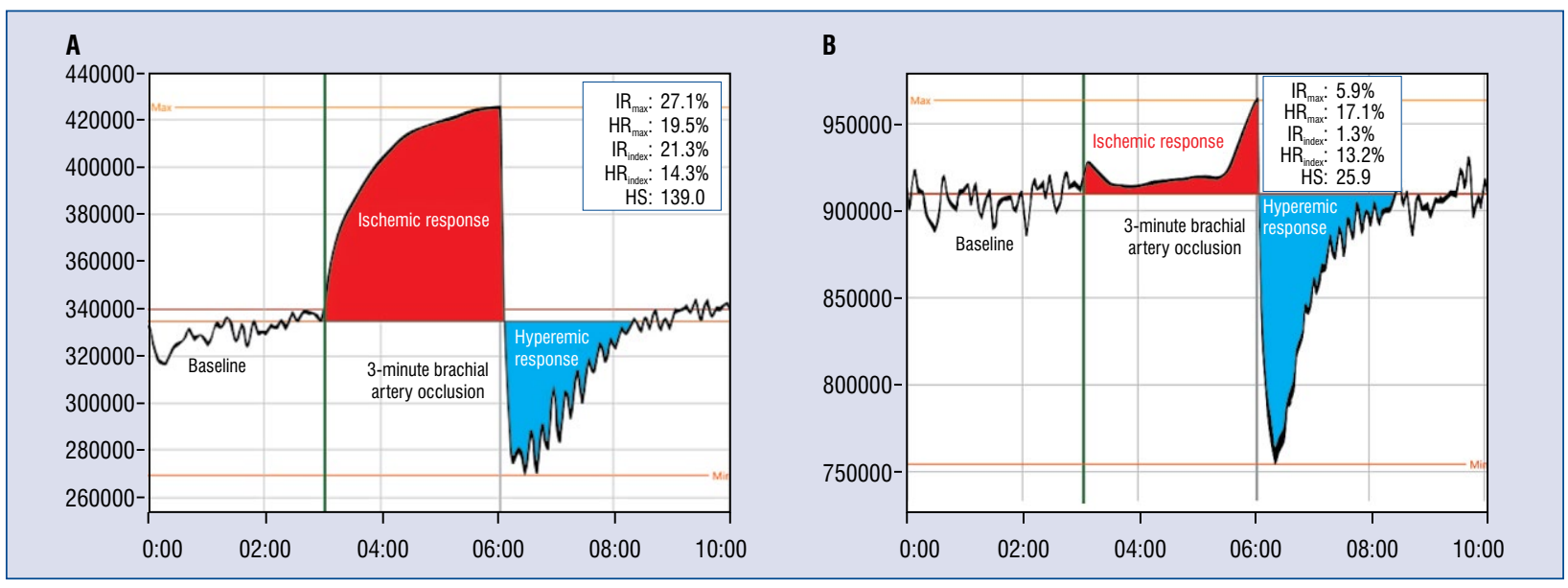

Figure 2. A. Typical image of nicotinamide adenine dinucleotide (NADH) fluorescence trace in a 30-year-old healthy subject with very high value of hypoxia sensitivity (HS) parameter as well as very dynamic ischemic (IR) and hyperemic responses (HR); B. Image of significant microvascular disturbances in NADH fluorescence trace in 30-year-old post-COVID patient with very low value of HS parameter as well as poor ischemic response $\left(I R_{\max }\right.$ and $\left.I R_{\text {index }}\right)$. 
the microcirculatory response to hypoxia. Interestingly, low HS values were related to a more severe course of COVID-19. Further, it was recently suggested that the microcirculatory response to hypoxia expressed as the HS parameter could be a prognostic factor in COVID-19 (Fig. 2A, B) [10].

Accumulating evidence suggests that we should consider COVID-19 as a systemic microvascular endothelial disease with different clinical manifestations from severe and acute to completely asymptomatic course [1,2]. Therefore, non-invasive, sensitive, and reliable methods for microvascular endothelial function clinical monitoring are strongly needed.

\section{Acknowledgments}

This work was supported by the European Union from the resources of the European Regional Development Fund under the Smart Growth Operational Program, Grant No. POIR. 01.01.01-00-0540/15.

\section{Conflict of interest: None declared}

\section{References}

1. Varga Z, Flammer AJ, Steiger P, et al. Endothelial cell infection and endotheliitis in COVID-19. Lancet. 2020; 395(10234): 1417-1418, doi: 10.1016/S0140-6736(20)30937-5, indexed in Pubmed: 32325026.

2. Gasecka A, Pruc M, Kukula K, et al. Post-COVID-19 heart syndrome. Cardiol J. 2021; 28(2): 353-354, doi: 10.5603/ CJ.a2021.0028, indexed in Pubmed: 33645626.
3. Lowenstein CJ, Solomon SD. Severe COVID-19 is a microvascular disease. Circulation. 2020; 142(17): 1609-1611, doi: 10.1161/CIRCULATIONAHA.120.050354, indexed in Pubmed: 32877231.

4. Østergaard L. SARS CoV-2 related microvascular damage and symptoms during and after COVID-19: Consequences of capillary transit-time changes, tissue hypoxia and inflammation. Physiol Rep. 2021; 9(3): e14726, doi: 10.14814/phy2.14726, indexed in Pubmed: 33523608.

5. Maccio U, Zinkernagel AS, Shambat SM, et al. SARS-CoV-2 leads to a small vessel endotheliitis in the heart. EBioMedicine. 2021; 63: 103182, doi: 10.1016/j.ebiom.2020.103182, indexed in Pubmed: 33422990.

6. Hellmann M, Roustit M, Cracowski JL. Skin microvascular endothelial function as a biomarker in cardiovascular diseases? Pharmacol Reports. 2015; 67(4): 803-810, doi: 10.1016/j. pharep.2015.05.008.

7. Katarzynska J, Lipinski Z, Cholewinski T, et al. Non-invasive evaluation of microcirculation and metabolic regulation using flow mediated skin fluorescence (FMSF): Technical aspects and methodology. Rev Sci Instruments. 2019; 90(10): 104104, doi: 10.1063/1.5092218

8. Tarnawska M, Dorniak K, Kaszubowski M, et al. A pilot study with flow mediated skin fluorescence: A novel device to assess microvascular endothelial function in coronary artery disease. Cardiol J. 2018; 25(1): 120-127, doi: 10.5603/CJ.a2017.0096, indexed in Pubmed: 28840593.

9. Hellmann M, Tarnawska M, Dudziak M, et al. Reproducibility of flow mediated skin fluorescence to assess microvascular function. Microvasc Res. 2017; 113: 60-64, doi: 10.1016/j. mvr.2017.05.004, indexed in Pubmed: 28529171.

10. Gebicki J, Katarzynska J, Marcinek A. Can efficient stimulation of myogenic microcirculatory oscillations by transient ischemia predict low incidence of COVID-19 infection? Respir Physiol Neurobiol. 2021; 286: 103618, doi: 10.1016/j.resp.2021.103618, indexed in Pubmed: 33460776. 